\title{
Some Characteristics of A Class of Edge-iteration Network Models Jing Su${ }^{1, a}$, Bing $\mathrm{Yao}^{2, \mathrm{~b}}{ }^{*}$ and Ming $\mathrm{Yao}^{3, \mathrm{c}}$
}

\author{
${ }^{1}$ College of Mathematics and Statistics, Northwest Normal University, Lanzhou, Gansu 730070, CHINA \\ ${ }^{2}$ College of Mathematics and Statistics, Northwest Normal University, Lanzhou, Gansu 730070, CHINA \\ ${ }^{3}$ Department of Information Process and Control Engineering, Lanzhou Petrochemical College of Vocational \\ Technology, Lanzhou, 730060, CHINA
}

A1099270659@qq.com, bCorresponding authors: yybb918@163.com, cyybm@163.com

Keywords: small-world networks; edge-iterations; collapse-rank.

\begin{abstract}
We present an example of deterministic network models in an iterative fashion. The network has small-world effect, scale-free topology and high degree of tolerance against random failure. We propose some new parameters for this model in order to further discuss the damage rate, such as collapse-rank, fragile-rank, as well as the edge-cumulative distribution and d-degree cumulative distribution, neighbor degree-average, attracting number. In addition, we compute these parameters for the model.
\end{abstract}

\section{Introduction}

In the past few years, a number of network models have been proposed to describe real-life complex networks, the most widely-studied models are the simple and attractive small-world network model made by Watts and Strogatz (WS model) and scale-free network model proposed by Barabasi and Albert (BA model), which triggered a sharp interest in the studies of the different properties of complex networks (Ref. [1], [2], [3], [4], [5], [6]). Obviously, the small-world effect and scale-free topology are much more general, moreover researchers have done a vast number of investigations to explore other mechanisms and properties of small-world networks.

Many complex systems display a surprising degree of tolerance against errors. For example, relatively simple organisms grow, persist and reproduce despite drastic damage deliberately or environmental interventions, an error tolerance attributed to the robustness of the underlying network, that is to say, although key components regularly malfunction local failure rarely lead to the collapse of the entire net-work (Ref. [7], [8], [9], [10]). In reality, some networks are extremely vulnerable to attacks, such as the World Wide Web, the airplanes connection networks, metabolic networks (Ref. [12]), social network-s,communications networks (Ref. [14]). So, it is necessary to study the damage degree and vulnerability degree of network for us.

As known, the main properties of scale-free networks that appear in the existing literature can be summarized as follows: scale-free networks have scaling (power law) degree distribution (Ref. [13]). Scale-free networks can be generated by certain random processes, the foremost among which is preferential attachment, scale-free networks have highly connected hubs that hold the network together and give the robust yet fragile feature of error tolerance but attack vulnerability (Ref. [13]). Small-world networks are characterized by three main properties (Ref. [15]). First, their average path length does not increase linearly with the system size, but grows with the number of vertices or slower. Second, average vertex degree of the network is small. Third, the network has a high average clustering (Ref. [1]) compared to an Erdos-Renyi (Ref. [2], [3]) random network of equal size and average vertex degree. According to the above properties, it have small average path, when the network is under attack, we can assume that whether it is damage is very big or not? How to picture a vertex being closely connected with its adjacent vertices? So, we put forward other researches to probe the nature of real-life complex networks.

Generation of Edge-iteration network models. In this section, we introduce a class of small-world and scale-free network models investigated in [15] also a similar model appeared in [11]. 
This model is constructed in an iterative fashion. We will calculate analytically some relevant properties for the models. We focus on the analysis of the functions and relations among the new properties in network models.

Iterative construction. We denote the network model after $\mathrm{t}$ step evolution by, and present the construction algorithm of $N(t)$ as follows (see for examples shown in Fig.1):

Algorithm 1 N-algorithm
Initialization. For $t=0, N(0)$ has three vertices and three edges connecting them to form a
triangle. Each edge of the triangle is defined as the generating edge.

Iteration. For $t \geq 1, N(t)$ is obtained from $N(t-1)$ by adding new vertices and edges. Do to every generating edge of $N(t-1)$, a new vertex is added, which is connected with the endpoints of the generating edge to form two generating edges of $N(t)$. The generating edges of $N(i)$ are not that of $N(t)$ if $i \leq t-1$.

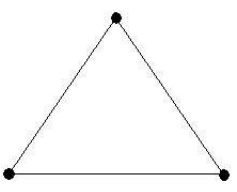

$t=0$

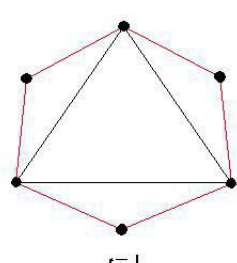

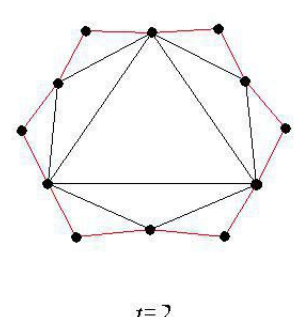

$t=2$

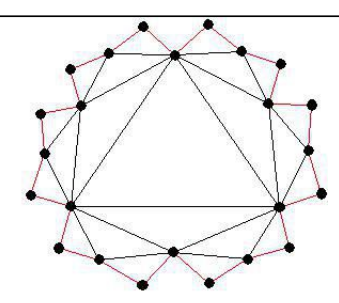

Figure 1: The construction of the network at the first four steps of the iterative process

Numbers of vertices and edges of $N(t)$. The number of edges of $N(t)$ is denoted by $n_{e}(t)$, the number of vertices of $N(t)$ is written as $n_{v}(t)$ at time step $t$ here after. In the evolution process of the model $N(t)$, for each new vertex added, two new edges are created. Therefore, the difference $\Delta n_{v}(t)=n_{v}(t)-n_{v}(t-1)=2 \Delta n_{v}(t-1)$ for $t>1$. Since $n_{v}(0)=3$ and $\Delta n_{v}(1)=3$, it follows that $\Delta n_{v}(t)=3 \cdot 2^{t-1}$, we have $n_{v}(t)=3 \cdot 2^{t}$. Hence, the difference $\Delta n_{e}(t)=n_{e}(t)-n_{e}(t-1)=2 \Delta n_{v}(t)=3 \cdot 2^{t}$. As $n_{e}(0)=3$, we can get $3 \cdot 2^{t+1}-3$. The average degree of $N(t)$ is then

$$
\langle k\rangle=\frac{2 n_{e}(t)}{n_{v}(t)}=\frac{2\left(3 \cdot 2^{t+1}-3\right)}{3 \cdot 2^{t}}=4\left(1-\frac{1}{2^{t+1}}\right)
$$

For large $t$ it is smaller and approximately equal to 4 . We can see that the model $N(t)$, when $t$ is large enough, is a sparse graph whose vertices have many fewer connections than is possible.

Topological properties of $N(t)$. We, in this section, focus on the topological behaviors of the model $N(t)$, such as the power law degree cumulative distribution, the clustering coefficient and the collapse-rank, fragile-rank, edge-cumulative distribution, d-degree cumulative distribution, neighbor degree-average, attracting number.

Degree cumulative distribution. The degree cumulative distribution is one of the most important statistical characteristics of a network. Notice that, the degree of a vertex $i$ is the number of edges incident from $i$, and we denoted by $k_{i}(t)$. The degree cumulative distribution $P_{c u m}(k)$ is the probability that vertex degree greater than or equal to $k$ in the network. By the $\mathrm{N}$-algorithm of $N(t)$, we have $k_{i}(t)=k_{i}(t-1)+2$, if $t_{c}$ is the step at which a vertex $i$ is created, then $k_{i}\left(t_{c}\right)=2$ and hence $k_{i}(t)=2\left(t-t_{c}+1\right)$. Therefore, the degree spectrum of $N(t)$ is a series of discrete values (Ref. [15]): at time step $t$, the number of vertices of degree $k=2,4,6, \ldots, 2(t-1), 2 t, 2(t+1)$, equals to $3 \cdot 2^{t-1}, 3 \cdot 2^{(t-1)-1}, \ldots$, $3 \cdot 2^{0}, 3$, respectively. Other values of degrees are absent in the degree spectrum. Due to the discreteness of this degree spectrum, as $0<\tau<t$, we obtain the cumulative degree distribution

$$
P_{\text {cum }}(k)=\sum_{i=0}^{\tau} n_{v}(i) / n_{v}(t)=\frac{n_{v}(0)+n_{v}(1)+\cdots+n_{v}(\tau-1)+n_{v}(\tau)}{n_{v}(t)}=\frac{2^{\tau+1}-1}{2^{t}} \propto 2^{\tau-t+1}
$$

Plug $\tau=t+1-1 / 2$ into the above form, we obtain $P_{\text {cum }}(k) \propto 2^{-k / 2}$. Obviously, when the size of the network is large, the cumulative degree distribution is an exponential power of degree $k$, so the 
model is exponential. If we regard that $t$ is continuous, so is $\tau$. Then we can take $\tau=t-1-(2 / \ln 2+1 / \ln 3) \ln k$, which leads to $P_{c u m}(k) \propto k^{-r_{c}}$, where $r_{c} \approx 2.6309$. We claim the model $N(t)$ obeys the power law distribution.

Clustering coefficient. Zhang et al.in [15] computed: As $t \rightarrow \infty,\langle C\rangle \propto \ln 2$, which approaches to a constant value 0.6931 , and so the clustering of the model is higher.

Collapse-rank and fragile-rank. The difficulty of repairing those destroyed networks depends on the key vertices of the model $N(t)$ if it was attacked. As a result, we design two new parameters to measure about the damage and the fragility of the networks (Ref. [8]). We only think of destroying the network situation can be divided into two kinds: first, removing the edge of the network; second, making the network collapse by destroying vertices. In general, the deletion of a subset $X \subset V(N(t))$ will destroy $N(t)$ to collapse. If a subset $X^{*} \subset V(N(t))$ makes the component number $\omega\left(N(t)-X^{*}\right) \geq \omega(N(t)-X)$ for any subset $X \subset V(N(t))$, we define $\operatorname{cor}(t)=\left|Y^{*}\right| /|V(N(t))|$ and $\operatorname{frr}(t)=\omega\left(N(t)-X^{*}\right) /|V(N(t))|$ to be the collapse-rank and the fragile-rank of $N(t)$, respectively. In our network model $N(t)$, we denote a vertex birth at step $i$ by $v_{i}$, we find the set $X^{*}=V_{0} \cup V_{1} \cup \cdots \cup V_{t-1}$ to be a key set, where $V_{i}$ is the set of the vertices birth at step $i$. Clearly, the component number $\omega\left(N(t)-X^{*}\right)=3 \cdot 2^{t-1}$ is maximal Hence, we get

$$
\operatorname{cor}(t)=\frac{\left|X^{*}\right|}{|V(N(t))|}=\frac{3 \cdot 2^{t-1}}{3 \cdot 2^{t}}=\frac{1}{2}, f r r(t)=\frac{\omega\left(N(t)-X^{*}\right)}{|V(N(t))|}=\frac{3 \cdot 2^{t-1}}{3 \cdot 2^{t}}=\frac{1}{2}
$$

For a malicious damage, the larger collapse-rank cor $(t)$ shows that the network was broken more seriously. The smaller fragile-rank $f r r(t)$ indicates that the network has more strong stability when facing attack.

Edge-collapse-rank and edge-fragile-rank. The deletion of a subset $Y \subset E(N(t))$ from $N(t)$ will make $N(t)$ collapse. If a subset $Y^{*} \subset E(N(t))$ makes the component number $\omega\left(N(t)-Y^{*}\right) \geq \omega(N(t)-Y)$ for any subset $Y \subset E(N(t))$, we define $\left|Y^{*}\right| /|E(N(t))|$ and $\omega\left(N(t)-Y^{*}\right) /|E(N(t))|$ to be the edge-collapse-rank and the edge-fragile-rank of $N(t)$. We use symbol $\operatorname{cor}_{E}(t)$ and $\operatorname{frr}_{E}(t)$ to mark them respectively. In the same way, we can also find meet the condition of $Y^{*}, Y^{*}=E(N(t))$, Clearly, the number of $\omega\left(N(t)-Y^{*}\right)=n_{v}(t)=3 \cdot 2^{t} \geq \omega(N(t)-E)$, for any $Y \subset E(N(t))$. Thus, $\left|Y^{*}\right|=n_{e}(t)=3 \cdot 2^{t+1}-3$, and $\operatorname{cor}_{E}(t)=1, \operatorname{frr}_{E}(t) \approx 1 / 2$.

The edge-cumulative and d-degree cumulative distributions. Motivated from the cumulative degree distribution that is an important character of scale-free networks the authors in [8] proposed a statistic way named as the d-cumulative distribution defined as $P_{d c u m}(k)=\sum d \cdot n_{d}\left(t_{i}\right) / \sum d \cdot n_{d}(t)$, where $n_{d}(k)$ is the number of vertices of degree $d$ at time step $t$. We found that the statistic is not exact and put forward another way defined as $P_{\text {ecum }}(k)=\sum_{i=0}^{\tau} n_{e}(i) / n_{e}(t)$ for $0<\tau<t$. According to the degree spectrum of the present network: at time $\mathrm{t} t$, the number of vertices of degree $k=2,4,6, \cdots, 2(t-1), 2 t, 2(t+1)$, equals respectively to $3 \cdot 2^{t-1}, 3 \cdot 2^{(t-1)-1}, 3 \cdot 2^{(t-2)-1}, \cdots, 3 \cdot 2^{0}, 3$, we have

$$
P_{\text {ecum }}(k)=\frac{1}{n_{e}(t)} \sum_{i=0}^{\tau} n_{e}(i)=\frac{3 \cdot 2^{0+1}-3+3 \cdot 2^{1+1}-3+\cdots+3 \cdot 2^{\tau-1+1}-3+3 \cdot 2^{\tau+1}-3}{3 \cdot 2^{t+1}-3} \propto 2^{\tau-t-1}
$$

So, $P_{\text {ecum }}(k) \sim P_{\text {cum }}(k)$ according to (2). We define a new index named as the degree cumulative distribution $P_{\text {cum }}^{d}(k)$. The specific expression is $P_{\text {aum }}^{d}(k)=\sum_{i=0}^{\tau} n_{d}(i) / n_{d}(t)$ for a fixed even integer $d \geq k$, 
where $n_{d}(t)$ is the number of vertices having degree $d$ and $n_{v}^{d}(i)=0$ if there are no vertices of degree $d$ in $N(i)$. Here, we have $P_{\text {cum }}^{d}(k)=\sum_{i=0}^{\tau} n_{d}(i) / n_{d}(t) \propto 2^{\tau-t+1}$. We have shown $P_{\text {cum }}^{d}(k) \sim P_{\text {cum }}(k)$.

Neighbor degree-average and attracting number. We define the neighbor degree-average $d_{\text {ave }}(x)$ of a vertex $x$ by $d_{\text {ave }}(x)=\sum_{y \in n i(x)} k(y, t) / k(x, t)$, where $k(u, t)$ is the degree of a vertex $u$ in $N(t)$, nei $(u)$ is the set of neighbors of the vertex $u$ in $N(t)$ (Ref. [8]). The attracting number of $x$ is defined as $\operatorname{att}(x)=(k(x, t)-1) / \sum_{y \in n e i(x)} k(y, t)$. It has been wanted that the attracting numbers of vertices follows the linear preferential attachment rule in scale-free networks. A vertex having higher attracting number means that vertex and its neighbors together can attract new vertices to form new edges at a new step time. In the following discussion for $x=v_{0}$, we have $d_{\text {ave }}\left(v_{0}\right) \cdot \operatorname{att}\left(v_{0}\right)=(2 t+1) / 2(t+1)$. when $x=v_{1}$, we obtain $d_{\text {ave }}\left(v_{1}\right) \cdot \operatorname{att}\left(v_{1}\right)=(2 t-1) / 2 t$, as $x=v_{i}(i \geq 2)$, we suppose $v_{j}$ is the one of the neighboring vertices before $i-2$ moment into the network, so, $0 \leq j \leq i-2, d_{\text {ave }}\left(v_{1}\right) \cdot \operatorname{att}\left(v_{1}\right)=(2 t-2 i+1) / 2[t-(i-1)]$.

\section{Conclusion}

In summary, we have studied a network model, built in an iterative fashion.As known, real-world networks have scale-free and small-world properties, therefore great effort is necessary in order to protect them from attacks. Researching the collapse of networks is more and more popular, but we have no detailed indicators to measure the extent of damage. So, the concepts of collapse-rank and fragile-rank are verified here. We also studied the neighbor degree-average and attracting numbers, two parameters can show the importance and position of a vertex in the whole network. Based on this view we can know which vertex is needed to be protected deliberately when the whole network is attacked. In addition, such a simple model explains how the malfunctioning of a single component of a real system can generate a cascading effect, thus causing the entire network to collapse (Ref. [16], [17], [18]). We believe that the model may help engineers in network topology-designing and performance analyzing, it may help to understand the small-world phenomenon and scale-free topology (Ref. [15]), it offers a guidance for us in further studying to protect real networks. Here, we propose a new parameter $P_{c u m}^{d}(k)$ and show $P_{\text {cum }}(k) \sim P_{\text {cum }}^{d}(k)$. As further investigation, we consider some generations of the above parameters on some general network models.

Acknowledgment. The second author, Bing Yao, thanks the National Natural Science Foundation of China under grants No. 61163054 and No. 61163037. The third author, MingYao, thanks The Special Funds of Finance Department of Gansu Province of China under grant No. 2014-63.

\section{References}

[1] M. Barthelemy and L. A. N. Amaral, phys. Rev. Lett. 82, 3180 (1999).

[2] M. E. J. Newman and D. J. Watts, Phys. Lett. A 263, 341 (1999).

[3] A. Barrat, and M. Weight, Eur. Phys. J. B 13, 547 (2000).

[4] L. A. N. Amaral, A. Scala, M. Barthelemy and H. E. Stanley, Proc. Natl. Acad. Sci. USA, 97, 11149 (2000).

[5] J. Kleinberg, Natune 406, 845 (2000).

[6] F. Comellas and M. Sampels, Physica A 309, 231 (2002).

S.N.D S. N. Dorogovtsev, A. V. Goltsev, J. F. F. Mendes, Phys. Rev. E 65 (2002) 066122.

[7] S. N. Dorogovtsev, A. V. Goltsev, J. F. F. Mendes, Phys. Rev. E 65 (2002) 066122. 
[8] Bing Yao, Hongyu Wang, Ming Yao, Xiang'en Chen, Chao Yang and Xiaomin Zhang. On The Collapse of Graphs Related to Scale-Free Networks. Proceeding of 2013 Third International Conference on Information Science and Technology. March 2013, Yangzhou, Jiangsu, China, 16-21.

[9] Bing Yao, Chao Yang, Ming Yao, Hongyu Wang, Xiang'en Chen, Xiaomin Zhang, Mogang Li. Graphs As Models of Scale-free Networks. 2013 International Conference on Information Technology and Computer Applications (ITCA 2013) 3680-3684.Applied Mechanics and Materials, Vol.380-384(2013) pp2034-2037. DOI: 10.4028/www.scientific.net/AMM.380-384.2034.

[10] Bing Yao, Xia Liu, Wan-jia Zhang, Xiang'en Chen, Xiao-min Zhang, Ming Yao, Zheng-xue Zhao. Applying Graph Theory To The Internet of Things. 2013 IEEE International Conference on High Performance Computing and Communications and 2013 IEEE International Conference on Embedded and Ubiquitous Computing, 2354-2361. DOI: 10.1109/HPCC.and.EUC.2013.339.

[11] Xia Liu, Bing Yao, Wanjia Zhang, Xiang'en Chen, Xinsheng Liu, Ming Yao. Uniformly Bound-Growing Network Models And Their Spanning Trees. The 2014 International Conference on Information and Communications Technologies (ICT2014), May 2014 in Nanjing, China. 2014, pp35-38.

[12] H. Jeong, B. Tombor, R. Albert, Z. N. Oltvai, A. -L. Barabasi, Nature 407 (2000)651.

[13] A. -L. Barab $\backslash^{\prime}\{a\}$ si, R. Albert, Science 286 (1999)509.

[14] R. Albert, H. Jeong, A. -L. Barabasi, Nature 401 (1999)130.

[15] Zhongzhi Zhang, Lili Rong, Chonghui Guo, A deterministic small-world network created by edge iterations. Phys. 363 \} (2006)567-572.

[16] P. Crucitti, V. Latora, M. Marchiori, A. Rapisarda,Physica A 320 (2003)622.

[17] P. Crucitti, V. Latora, M. Marchiori, cond-mat/0309141 and Phys. Rev. E 69 (2004) 045104 (R).

[18] V. Latora, M. Marchiori, Phys. Rev. Lett. 87 (2001)198701. 